\title{
Review
}

\section{Glycosylation in Congenital Muscular Dystrophies}

\author{
Tamao ENDO ${ }^{*, a}$ and Tatsushi TodA ${ }^{b}$ \\ ${ }^{a}$ Glycobiology Research Group, Tokyo Metropolitan Institute of Gerontology, Foundation for Research on Aging and \\ Promotion of Human Welfare; 35-2 Sakaecho, Itabashi-ku, Tokyo 173-0015, Japan: and \\ ${ }^{b}$ Division of Functional Genomics, Department of Post-Genomics and Diseases, \\ Osaka University Graduate School of Medicine; 2-2-B9 Yamadaoka, \\ Suita, Osaka 565-0871, Japan. \\ Received September 30, 2003
}

\begin{abstract}
Mammalian cells produce many glycoproteins, i.e., proteins with covalently attached sugar chains. Recent advances in glycobiology have revealed the importance of sugar chains as biosignals for multi-cellular organisms including cell-cell communication, intracellular signaling, protein folding, and targeting of proteins within cells. The $O$-mannosyl linkage, which used to be considered specific to yeast, has recently been found in mammals. One of the best known $O$-mannosyl-modified glycoproteins is $\alpha$-dystroglycan, which is a central component of the dystrophin-glycoprotein complex isolated from skeletal muscle membranes. We have identified and characterized a glycosyltransferase, UDP- $N$-acetylglucosamine: protein $O$-mannose $\beta 1,2-N$-acetylglucosaminyltransferase (POMGnT1), involved in the biosynthesis of $O$-mannosyl glycans. We subsequently found that loss of function of the POMGnT1 gene is responsible for muscle-eye-brain disease (MEB). MEB is an autosomal recessive disorder characterized by congenital muscular dystrophy, ocular abnormalities and brain malformation (type II lissencephaly). Moreover, recent data suggest that aberrant protein glycosylation of $\alpha$-dystroglycan is the primary cause of some forms of congenital muscular dystrophy. Here we review new insights into the glycobiology of muscular dystrophy and neuronal migration disorder.
\end{abstract}

Key words glycobiology; $O$-mannosylation; muscular dystrophy; dystroglycan; glycosyltransferase; muscle-eye-brain disease

Now that sequencing of the human genome is essentially complete, a new era of postgenomic research has begun and is expected to result in revolutionary developments in medical science. Because over $60 \%$ of the proteins produced by the human body are thought to contain sugar chains, many important problems in life science will fall in the field of glycobiology. Unlike proteins and nucleic acids, which are linear molecules, sugar chains are branched and the number of isomers (linkage isomers, $1-2,1-3,1-4,1-6,2-3,2-6$, and $2-8)$ and anomeric configurations $(\alpha$ and $\beta$ ) increases geometrically with size. Thus, a specific characteristic of sugar chains, i.e., one not shared by nucleic acids and proteins, is that they can form many possible structures with a small number of units. Because of the difficulties in determining the structures of sugar chains of glycoproteins, many of the functional aspects of the sugar moieties were ignored during the long history of protein research. However, newly developed and sensitive methods to elucidate the structures and functions of the sugar chains have opened the way to investigate these molecules. Recent data have revealed the importance of sugar chains as biosignals for multi-cellular organisms including cell-cell communication, intracellular signaling, protein folding, and targeting of proteins within cells. ${ }^{1,2}$ The biosynthesis of sugar chains is not formed by the intervention of a template but is under the control of the expression of glycosyltransferases, their substrate specificity, and their localization in specific tissues. There is growing evidence that these enzymes have a variety of roles in cellular differentiation and development, as well as in disease processes.

The major sugar chains of glycoproteins can be classified into two groups according to their sugar-peptide linkage regions. Those that are linked to asparagine (Asn) residues of proteins are termed $\mathrm{N}$-glycans, while those that are linked to serine (Ser) or threonine (Thr) residues are called $O$-glycans.
In $N$-glycans, the reducing terminal $N$-acetylglucosamine (GlcNAc) is linked to the amide group of Asn via an aspartylglycosylamine linkage. In $O$-glycans, the reducing terminal $N$-acetylgalactosamine (GalNAc) is attached to the hydroxyl group of Ser and Thr residues. In addition to the abundant $O$-GalNAc forms, several unique types of protein $O$-glycosylation have been found, such as $O$-linked fucose, glucose, GlcNAc, and mannose, which have been shown to mediate diverse physiological functions. For example, $O$ mannosylation has recently been shown to be important in muscle and brain development.

Muscular dystrophies are genetic diseases that cause progressive muscle weakness and wasting. ${ }^{3)}$ The causative genes of several muscular dystrophies have been identified in the past fifteen years. ${ }^{4}$ The best known is the one described by Duchenne that results from mutations in the gene encoding a protein called dystrophin. Another subclass is congenital muscular dystrophies, where muscle weakness is apparent at birth or shortly afterwards. Recent data suggest that aberrant glycosylation of the glycoprotein $\alpha$-dystroglycan is the primary cause of some forms of congenital muscular dystrophy. This article reviews new insights into the glycobiology of muscular dystrophy and neuronal migration disorder.

Structures of $\boldsymbol{O}$-Mannosyl Glycans $O$-Mannosylation is known as a yeast-type modification, and $O$-mannosylated glycoproteins are abundant in the yeast cell wall. ${ }^{5)}$ In unicellular eukaryotic organisms, all $O$-mannosyl glycan structures elucidated so far are neutral linear glycans consisting of 1 to 7 mannose residues..$^{5)} O$-Mannosylation of proteins has been shown to be vital in yeast, and its absence may affect cell wall structure and rigidity. Additionally, a deficiency in protein $O$-mannosylation in the fungal pathogen Candida albicans leads to defects in multiple cellular functions including expression of virulence. ${ }^{6)}$ In addition to fungi and yeast, clam worm has an $O$-mannosyl glycan (a glucuronosyl $\alpha 1-6$ man- 
nosyl disaccharide) in skin collagen. ${ }^{7)}$ In mammals, a brain proteoglycan was the first $O$-mannosylated protein to be identified. $^{8-10)}$ It was found to be a sialylated oligosaccharide with the structure Neu5Ac $\alpha 2-3 \mathrm{Gal} \beta 1-4 \mathrm{GlcNAc} \beta 1-3 \mathrm{Man}-$ Ser/Thr. Other mammalian $O$-mannosyl glycans include Gal $\beta 1$-4GlcNAc $\beta 1$-3Man-Ser/Thr, GlcNAc $\beta 1-3$ Man-Ser/ Thr, Man-Ser/Thr, Gal $\beta 1-4($ Fuc $\alpha 1-3)$ GlcNAc $\beta 1-3$ Man$\mathrm{Ser} / \mathrm{Thr}$, and an unidentified sulfated $O$-mannosyl glycan.

However, we previously concluded that the major sialylated $O$-linked oligosaccharide of the bovine peripheral nerve $\alpha$-dystroglycan has the structure Neu5Ac $\alpha 2-3 \mathrm{Gal} \beta 1-4 \mathrm{Glc}-$ NAc $\beta 1-2$ Man-Ser/Thr. ${ }^{11)}$ This structure is similar to the one reported for mammalian brain proteoglycan, ${ }^{8-10)}$ except that it has a different linkage between the $\mathrm{N}$-acetylglucosamine and the mannose residues, 3-substituted mannose and 2-substituted mannose. Interestingly, we found the same $O$-mannosyl glycan in rabbit skeletal muscle $\alpha$-dystroglycan. ${ }^{12)} \mathrm{Be}-$ cause glycosylation of proteins is often both tissue- and species-dependent, ${ }^{1,2)}$ the presence of this unique $O$-mannosyl glycan in $\alpha$-dystroglycans from different tissues of different species raises the possibility that it mediates the important biological roles of $\alpha$-dystroglycan (described below). In addition to the $O$-mannosyl glycan described above, we found almost equal amounts of the core 1 structure, $\operatorname{Gal} \beta 1$ 3 GalNAc, in rabbit skeletal muscle $\alpha$-dystroglycan. ${ }^{12)}$ This is consistent with previous reports that desialylated rabbit skeletal muscle $\alpha$-dystroglycan reacted with peanut agglutinin, which is known to bind specifically with the Gal $\beta 1$ 3GalNAc group. ${ }^{13)}$

After our reports of the sialylated oligosaccharide with the $O$-mannosyl linkage, ${ }^{11,12)}$ an HNK-1 epitope (sulfoglucuronyl lactosamine) carrying $O$-mannosyl glycans $\left(\mathrm{HSO}_{3}-3 \mathrm{GlcA} \beta 1\right.$ $3 \mathrm{Gal} \beta 1-4 \mathrm{GlcNAc} \beta 1-2 \mathrm{Man}-\mathrm{Ser} / \mathrm{Thr}$ ) was detected in total brain glycopeptides. ${ }^{14)}$ It is noteworthy that these oligosaccharides have not only 2-substituted mannose but also 2,6disubstituted mannose. ${ }^{15)}$ Later, Smalheiser et al. showed ${ }^{16)}$ that dystroglycan from sheep brain carried the Neu5Ac $\alpha 2$ 3Gal $\beta 1$-4GlcNAc $\beta 1-2 \mathrm{Man}$, Gal $\beta 1-4 \mathrm{GlcNAc} \beta 1-2 \mathrm{Man}$, and Gal $\beta 1-4(F u c \alpha 1-3)$ GlcNAc $\beta 1-2$ Man structures. Mouse
$\mathrm{J} 1 /$ tenascin, which is involved in neuron-astrocyte adhesion, also contains these $O$-mannosyl glycans. ${ }^{17}$ ) Therefore, it is likely that a series of $O$-mannosyl glycans, with heterogeneity of mannose-branching and peripheral structures, is present in mammals. ${ }^{18)}$ Further studies are needed to clarify the distribution of such $O$-mannosyl glycans in various tissues and to examine their changes during development and under pathological conditions.

A comment must be added concerning the presence of 3substituted mannose in the original studies on brain chondroitin sulfate proteoglycans. ${ }^{8-10)}$ Their mass spectrum data indicated that the substitution to mannose was either at the C-2 position or C-3 position. Based on fragment patterns of trimethysilyl derivatives of deuterated disaccharides, they concluded that a 2-substitution was unlikely. However, a recent study suggests a rearrangement occurred when the sample was deuterated. ${ }^{14)}$ Therefore, it seems likely that brain proteoglycans, like other reported glycoproteins, contain 2substituted mannose.

Dystroglycan and Its Sugar Chains Dystroglycan is encoded by a single gene $(D A G 1)$ and is cleaved into two proteins, $\alpha$-dystroglycan and $\beta$-dystroglycan, by posttranslational processing. ${ }^{19)}$ In skeletal muscle, dystroglycan is a central component of the dystrophin-glycoprotein complex (DGC) (Fig. 1). $\alpha$-Dystroglycan is a heavily glycosylated extracellular peripheral membrane glycoprotein that anchors to the cell membrane by binding to a transmembrane glycoprotein, $\beta$-dystroglycan. The $\alpha$-dystroglycan- $\beta$-dystroglycan complex is expressed in a broad array of tissues and is thought to stabilize the plasma membrane by acting as an axis through which the extracellular matrix is tightly linked to the cytoskeleton. This is because $\alpha$-dystroglycan strongly binds to extracellular matrix proteins containing LamG domains, such as laminin, neurexin, agrin, and perlecan in a calcium-dependent manner. ${ }^{20)}$ The DGC contains a sarcoglycan complex. On the other hand, the cytoplasmic domain of $\beta$-dystroglycan contains a PPXY motif that interacts with dystrophin, which in turn binds to the actin cytoskeleton, syntrophins and $\alpha$-dystrobrevin. $\alpha$-Syntrophin and $\alpha$-dystro-

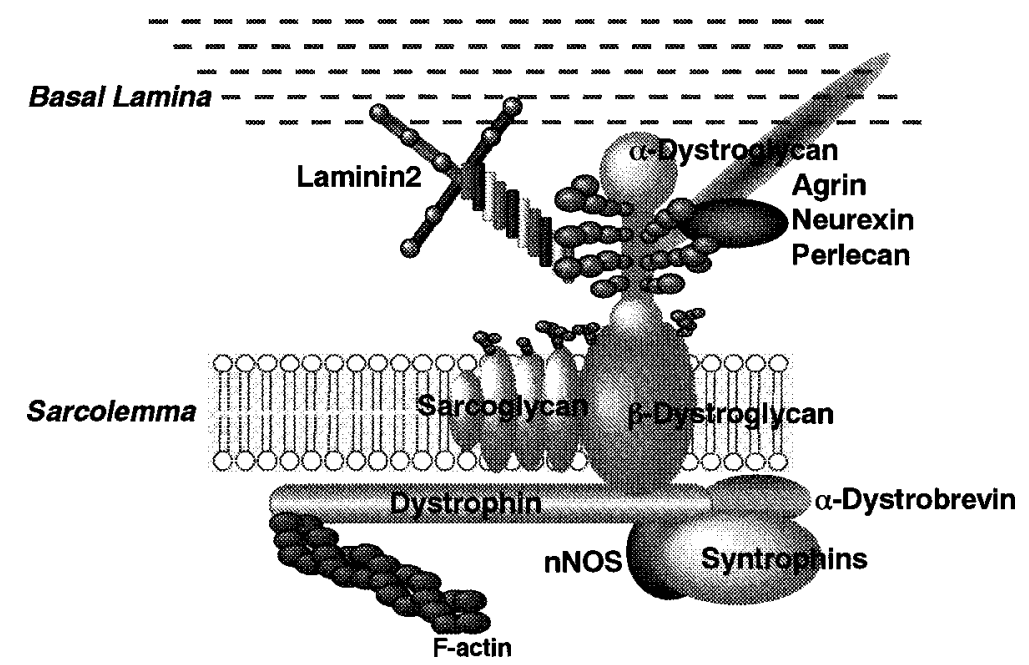

Fig. 1. Dystrophin-Glycoprotein complex (DGC) and Linkage between Laminin 2 in the Extracellular Matrix and Actin in the Subsarcolemmal Cytoskeleton

$\alpha$-Dystroglycan is a key component of the DGC and is modified by $O$-mannosyl glycan and binds to laminin 2 via its glycan. $\alpha$-Dystroglycan is also known to bind to other extracellular matrix proteins containing LamG domains, such as neurexin, agrin and perlecan. On the other hand, inside the cell, $\beta$-dystroglycan is known to bind to several components directly or indirectly. nNOS, neural NO synthetase. 
brevin can interact with neural NO synthetase (nNOS). Based on this molecular organization, the DGC is thought to contribute to the structural stability of the muscle cell membrane during cycles of contraction and relaxation. In human, mutations in dystrophin cause Duchenne and Becker muscular dystrophy, mutations in sarcoglycan cause limb-girdle muscular dystrophy, and mutations in laminin $\alpha 2$ chain cause congenital muscular dystrophy. ${ }^{4)}$

The function of dystroglycan in the body has been examined by targeting the $D A G 1$ gene in mice. However, disruption of this gene in mice is embryonically lethal. ${ }^{21)}$ Analysis of the mutant phenotype reveals a disruption of Reichert's membrane, an extra-embryonic basement membrane that separates the embryo from the maternal circulation. To allow the embryo to develop, chimeric mice generated from targeted embryonic stem cells have been produced. Dystroglycan-null chimeric mice showed muscular dystrophy, although muscle basement membrane formation was normal. ${ }^{22}$ The function of dystroglycan in specific tissues was examined with cre-LoxP technology. Targeting the dystroglycan gene specifically in differentiated skeletal muscle did not affect muscle basement membrane formation but resulted in a mild dystrophic phenotype. ${ }^{23)}$ Targeting the dystroglycan gene in brain resulted in abnormal cerebral cortical layering resembling human cobblestone lissencephaly, and defects in cerebellar granule cell migration. ${ }^{24}$ Targeting the dystroglycan gene in peripheral nerves caused defects in both myelination and nodal architecture. ${ }^{25)}$ These results indicate that dystroglycan is essential for normal development. Recent studies suggest that not only dystroglycan itself but also the attached sugars are important. The binding of $\alpha$-dystroglycan to extracellular matrix proteins requires the participation of its sugars, which are probably $O$-mannosyl glycans. ${ }^{26)}$ We also find that the sialyl $O$-mannosyl glycan (Neu5Ac $\alpha 2-3 \mathrm{Gal} \beta 1-4 \mathrm{Glc}-$ NAc $\beta 1$-2Man) is a laminin-binding ligand of $\alpha$-dystroglycan. ${ }^{11}$ Thus, mammalian $O$-mannosyl glycans may mediate the important biological roles of $\alpha$-dystroglycan.

Identification and characterization of the enzymes involved in the biosynthesis of mammalian type $O$-mannosyl glycans will help to elucidate the function and regulation of expression of these glycans. A key difference between mammalian and yeast-type $O$-mannosyl glycans is that those in mammals have the GlcNAc $\beta 1$-2Man linkage (Fig. 2), as de- scribed above. This linkage is assumed to be catalyzed by a glycosyltransferase, UDP- $N$-acetylglucosamine: protein $O$ mannose $\beta 1,2-N$-acetylglucosaminyltransferase (POMGnT1). POMGnT1 catalyzes the transfer of $N$-acetylglucosamine from UDP-GlcNAc to $O$-mannosyl glycoproteins. After we developed an enzyme assay for POMGnT1, its activity was found in brain homogenates of several mammals. ${ }^{27)}$ It should be noted that GlcNAc $\beta 1$-2Man linkages are also found in $N$-glycans, where they are catalyzed by two enzymes, UDP$N$-acetylglucosamine: $\alpha$-3-D-mannoside $\beta-1,2-N$-acetylglucosaminyltransferase I (GnT-I) and UDP- $N$-acetylglucosamine: $\alpha$-6-D-mannoside $\beta-1,2-N$-acetylglucosaminyltransferase II (GnT-II). However, we found that recombinant GnTI and GnT-II had no ability to catalyze the GlcNAc $\beta 1-2 \mathrm{Man}$ linkage in $O$-mannosyl glycans, ${ }^{27)}$ suggesting that a new enzyme must be responsible for the formation of this linkage. Thus, we cloned the human POMGnT1 gene on the basis of human cDNA sequences homologous to human GnT-I. ${ }^{28)}$ The nucleotide sequence indicated that POMGnT1 is a 660 amino acid protein with a calculated molecular mass of $71.5 \mathrm{kDa}$. A hydrophobicity analysis and secondary structure prediction of the amino acid sequence suggested that human POMGnT1 is a type II membrane protein. This topology was similar to the topologies of other Golgi glycosyltransferases.

Careful examination of substrate specificity of POMGnT1 indicated that recombinant POMGnT1 did not have either GnT-I or GnT-II activity. ${ }^{28)}$ As described above, GnT-I and GnT-II did not have any POMGnT1 activity. Taken together, these results suggest that loss-of-function of POMGnT1 is not compensated by GnT-I and GnT-II. Mammals are known to have an absolute requirement for GnT-I during early embryogenesis. Mouse embryos lacking a functional GnT-I gene die prenatally at E9.5 with multisystemic abnormalities. ${ }^{29,30)}$ On the other hand, over $60 \%$ of mouse embryos with null mutations in the GnT-II gene survive to term, but $99 \%$ of newborns die during the first week of postnatal development with multisystemic abnormalities. ${ }^{31)}$ Furthermore, a human disease caused by mutations in the GnT-II gene is known as congenital disorders of glycosylation type IIa [CDG-IIa: OMIM 212066, OMIM=Online Mendelian Inheritance in Man (http://www.ncbi.nih.gov/)]. CDG will be described later. Patients with CDG-IIa showed hypotonia, severe psychomotor retardation, frequent infections, and widely spaced

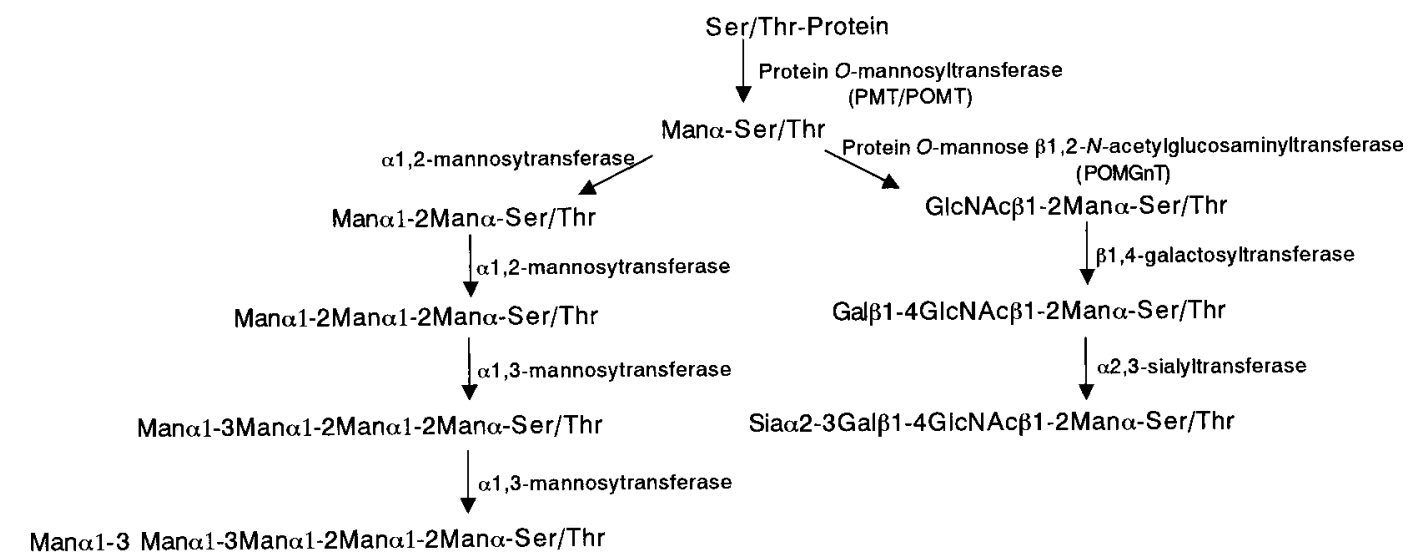

yeast

mammals

Fig. 2. Biosynthetic Pathway of Yeast $O$-Mannosyl Glycan and Proposed Biosynthetic Pathway of Mammalian $O$-Mannosyl Glycan 
nipples. ${ }^{32)}$ No human diseases having defects in GnT-I have been reported, suggesting that such defects are embryonically lethal and that GnT-I is essential for normal human development.

Identification of the Gene Responsible for Muscle-EyeBrain Disease (MEB) The human POMGnTl gene is located at $1 \mathrm{p} 33$, within the small candidate interval for muscleeye-brain disease (MEB). MEB is an autosomal recessive disorder characterized by congenital muscular dystrophy, ocular abnormalities and brain malformation (type II lissencephaly). ${ }^{33)}$ Patients with MEB show severe cerebral and ocular anomalies, but some patients reach adulthood. MEB has been observed mainly in Finland. Since defects of DGC cause muscular dystrophies ${ }^{4)}$ and $O$-mannosyl type glycan is required for the laminin binding of $\alpha$-dystroglycan in DGC, ${ }^{11)}$ it is possible that mutations in the POMGnTl gene are related to MEB.

To test this hypothesis, we screened the entire coding region and the exon/intron flanking sequences of the POMGnT1 gene for mutations in patients with MEB. We identified six independent disease-causing mutations in these patients. ${ }^{28)}$ Later we found seven more mutations for a total of 13 disease-causing mutations in patients with $\mathrm{MEB}^{34}$ ) (Fig. 3). We have not detected these 13 substitutions in any of 300 normal chromosomes, indicating that they are pathogenic and that the POMGnT1 gene is responsible for MEB. To confirm that the mutations observed in patients with MEB are responsible for the defects in the synthesis of $O$-mannosyl glycan, we expressed all of the mutant proteins and found that none of them had enzymatic activity. ${ }^{28,35)}$ Together, these findings indicate that MEB is inherited as a loss-of-function of the POMGnT1 gene. If POMGnT1 does not work, no peripheral structure (Neu5Ac $\alpha 2-3 \mathrm{Gal} \beta 1-4 \mathrm{GlcNAc}, \mathrm{Gal} \beta 1$ -
4(Fuc $\alpha 1-3)$ GlcNAc, and $\left.\mathrm{HSO}_{3}-3 \mathrm{GlcA} \beta 1-3 \mathrm{Gal} \beta 1-4 \mathrm{GlcNAc}\right)$ can be formed on $O$-mannosyl glycans. Because these structures are involved in adhesive processes, a defect of $O$-mannosyl glycan may severely affect cell migration and cell adhesion. Additionally, we found a selective deficiency of $\alpha$ dystroglycan in MEB patients. ${ }^{36}$ ) This finding suggests that $\alpha$-dystroglycan is a potential target of POMGnT1 and that hypoglycosylation of $\alpha$-dystroglycan may be a pathomechanism of MEB. MEB muscle and brain phenotypes can be explained by a loss of function of $\alpha$-dystroglycan due to abnormal $O$-mannosylation.

Aberrant Glycosylation of $\alpha$-Dystroglycan in Other Muscular Dystrophies The results of recent investigations suggest that some muscular dystrophies are caused by abnormal glycosylation of $\alpha$-dystroglycan, e.g., Fukuyama-type congenital muscular dystrophy (FCMD: OMIM 253800), congenital muscular dystrophy type 1C (MDC1C: OMIM 606612), Walker-Warburg syndrome (WWS: OMIM 236670 ) and the myodystrophy (myd) mouse (Table 1). However, the details of the defects are still unclear. Identification of these defects may provide new clues to the glycopathomechanism of muscular dystrophy.

Like MEB, FCMD and WWS are autosomal recessive disorders that are characterized by congenital muscular dystrophy, lissencephaly, and eye anomalies. ${ }^{37,38)}$ FCMD is a relatively common autosomal recessive disorder in the Japanese population. ${ }^{37)}$ It is the second most common form of childhood muscular dystrophy in Japan after Duchenne muscular dystrophy. Based on an average incidence of 3 per 100000 population, one in $c a$. 90 persons could be a heterozygous carrier in Japan. Kobayashi et al. ${ }^{39)}$ identified a gene on chromosome 9q31 that is responsible for FCMD. The gene encodes a novel 461-amino-acid protein of unknown function.

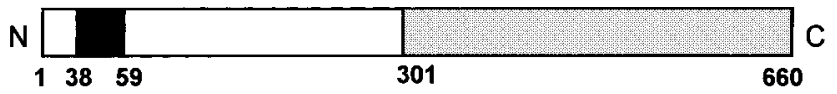

\begin{tabular}{|c|l|l|}
\hline & \multicolumn{1}{|c|}{ Mutation } & \multicolumn{1}{|c|}{ Effect } \\
\hline 1 & $281 \mathrm{C}>\mathrm{T}$ & Arg63Stop Nonsense \\
\hline 2 & 541 del T & Phe149 frameshift 167Stop \\
\hline 3 & $761 \mathrm{G}>$ A & Glu223 Lys Missense \\
\hline 4 & $900 \mathrm{G}>$ A & Cys269 Tyr Missense \\
\hline 5 & 1077 ins G & Val328 frameshift 338Stop \\
\hline 6 & 1106 ins T & Asp338 frameshift 338 Stop \\
\hline 7 & 1572 C $>$ G & Pro493 Arg Missense \\
\hline 8 & IVS17+1 G > A & Glu514 read-through 526Stop \\
9 & IVS17+1 G > T & /Leu472-His513 del \\
\hline 10 & 1743 G $>$ A & Ser536-Ser550 del \\
\hline 11 & 1813 del C & His573 frameshift 633Stop \\
\hline 12 & 1926 del T & Leu611 frameshift 633Stop \\
\hline 13 & 1970 del G & Val626 frameshift 633Stop \\
\hline
\end{tabular}

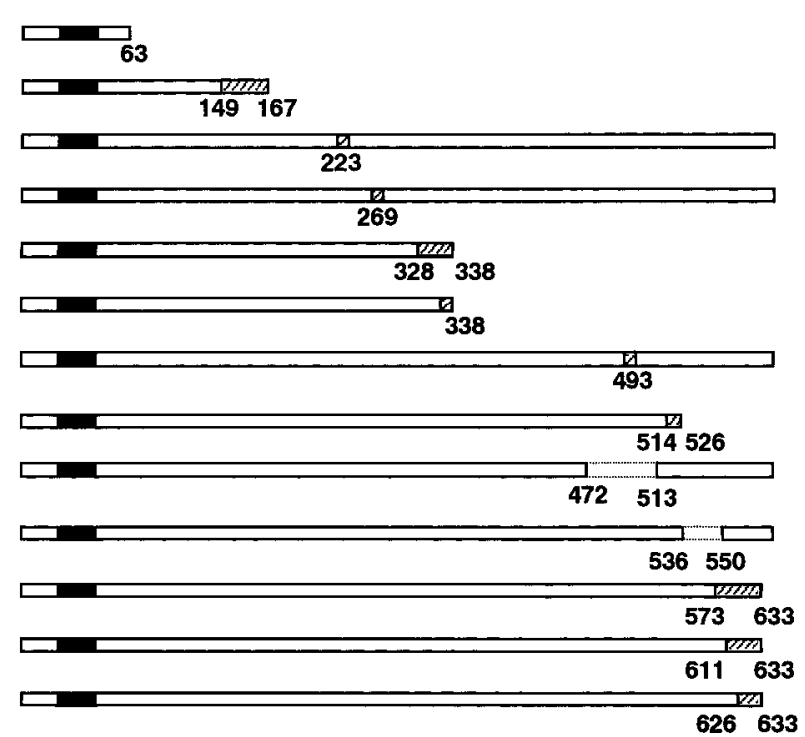

Fig. 3. Schematic Representation of POMGnT1 and the Predicted Products Corresponding to Each Mutation, and a Summary of Mutations of MEB Patients

Each protein is represented by a box with its $\mathrm{N}$ terminus to the left. Each box from the left indicates a cytoplasmic tail (white box), a transmembrane domain (black box), a stem domain (white box), and the catalytic domain (gray box). The hatched boxes and dotted-line boxes represent mutated amino acids and deletion regions, respectively. The numbers below the boxes indicate the amino acid residue numbers of POMGnT1 and each mutant. 
The protein, named fukutin because of its association with FCMD, has an N-terminal hydrophobic region suggesting a signal sequence or a transmembrane domain. A sequence analysis predicts it to be an enzyme that is involved in glycosylation. ${ }^{40)}$ Consistent with this finding, highly glycosylated $\alpha$-dystroglycan was found to be selectively deficient in the skeletal muscle of FCMD patients. ${ }^{41)}$ Recently, Takeda et $a l^{42)}$ generated chimeric mice using embryonic stem cells in which the fukutin gene was targeted for disruption. These mice developed severe muscular dystrophy, with a selective deficiency of $\alpha$-dystroglycan and its laminin-binding activity. These mice also had central nervous and ocular abnormalities. Taken together, these results indicate that fukutin is necessary for the maintenance of muscle integrity, cortical histogenesis and normal ocular development, and suggest a functional linkage between fukutin and $\alpha$-dystroglycan.

WWS is another form of congenital muscular dystrophy that is characterized by severe brain malformation (type II lissencephaly) and eye involvement. ${ }^{38)}$ Patients with WWS are severely affected from birth and usually die within their first year. WWS has a worldwide distribution. Recently, 20\% of WWS patients (6 of 30 unrelated WWS cases) have been found to have mutations in protein $O$-mannosyltransferase 1 (POMT1), a putative $O$-mannosyltransferase that catalyzes the transfer of mannose to a Ser or Thr residue on the basis of homology with the seven yeast $O$-mannosyltransferases. ${ }^{43}$ POMT1 is highly expressed in fetal brain, testis and skeletal muscle, which are the tissues that are affected in WWS. It is noteworthy that none of the 30 cases studied had mutations in another homologue, POMT2. This suggests that other as yet unidentified genes are responsible for this syndrome. However, it is unclear whether the POMT1 and POMT2 proteins actually catalyze $O$-mannosylation. ${ }^{43,44)}$ So far, attempts to detect protein $O$-mannosyltransferase activity of POMTs in vertebrates have not been successful. In WWS patients, as in MEB and FCMD patients, a highly glycosylated $\alpha$-dystroglycan was selectively deficient in skeletal muscle, while $\beta$ dystroglycan and laminin $\alpha 2$ were still expressed. Interestingly, the $r t$ mutant in Drosophila showed defects of myogenesis caused by a homologue of POMT1. ${ }^{45)}$ Although the $r t$ gene product is not known to initiate the biosynthesis of $O$ mannosyl glycan, $O$-mannosylation may be essential for muscular development in both vertebrates and invertebrates.

Defective glycosylation of $\alpha$-dystroglycan has also been implicated in congenital muscular dystrophy type 1C (MDC1C), which is caused by a homologue of fukutin (fukutin-related protein, $F K R P$ ). ${ }^{46}$ MDC1C is characterized by severe muscle weakness and degeneration, and cardiomyopathy. Mental retardation and cerebellar cysts have been observed in some forms. Allelic mutations in the FKRP gene also cause a milder and more common form of muscular dystrophy called limb-girdle muscular dystrophy 2I (LGMD2I: OMIM 607155), which is frequently associated with cardiomyopathy and which has a variable onset ranging from adolescence to adulthood. ${ }^{47)}$ Patients with mutations in the FKRP gene invariably have a reduced expression of $\alpha$-dystroglycan, which is strongly correlated with disease severity. A western blot analysis shows an apparent loss of higher molecular weight forms of $\alpha$-dystroglycan. Although the function of FKRP is unknown, it is suggested that FKRP is involved in the glycosylation of $\alpha$-dystroglycan as a glyco- syltransferase or a kind of modulator. Because FKRP and fukutin are thought to be Golgi-resident proteins ${ }^{48)}$ it is possible that defects of these proteins cause abnormal processing of $\alpha$-dystroglycan.

Finally, the gene large, which is mutated in the myodystrophy (myd) mouse, encodes a putative glycosyltransferase. ${ }^{49}$ ) However, its biochemical activity has not yet been confirmed. The causative mutation in myd was identified as a deletion of exons 5-7 of the large gene. This deletion results in a frameshift in the corresponding mRNA, leading to a premature termination codon. The myd mouse shows a progressive muscular dystrophy, ocular defects, and a central nervous system phenotype characterized by abnormal neuronal migration in the cerebral cortex, cerebellum and hippocampus, and disruption of the basal lamina. ${ }^{26,50)}$ The myd mouse, like MEB and FCMD patients, showed hypoglycosylation of $\alpha$ dystroglycan in muscle and brain. The human homologue of the large gene (LARGE) may be involved in novel forms of muscular dystrophy. A recent study described a patient with congenital muscular dystrophy, profound mental retardation, white matter changes and subtle structural abnormalities in the brain and a reduction of immunolabelling of $\alpha$-dystroglycan. ${ }^{51)}$ This patient was found to have a missense mutation and a 1 bp insertion in the $L A R G E$ gene.

Congenital Disorders of Glycosylation Defects in glycosylation pathways that are responsible for human diseases were initially identified in 1980 . Since then, at least 13 such defects, called congenital disorders of glycosylation (CDGs), have been identified. ${ }^{52)}$ The CDGs are a group of inherited multisystemic disorders, which are commonly associated with severe psychomotor and mental retardation. The characteristic biochemical feature of CDGs is the defective glycosylation of glycoproteins due to mutations in genes required for the biosynthesis of $\mathrm{N}$-glycans. CDG type I is caused by defects of the assembly of lipid-linked oligosaccharides, whereas CDG type II is caused by all defects of trimming and elongating of $\mathrm{N}$-glycans. $\mathrm{N}$-Glycosylation defects are routinely detected by isoelectric focusing of serum transferrin, which normally carries two-sialylated biantennary $\mathrm{N}$-glycans. The hyposialylated transferrin from CDG patients shows a cathodic shift, which in CDG-I is due to the loss of either one or both $\mathrm{N}$-glycans, and in CDG-II is due to the incomplete processing of protein-bound $\mathrm{N}$-glycans. The molecular nature of nine CDG-I types and four CDG-II types have been identified, and several will be described here briefly. By far the most common type, CDG-Ia (OMIM 212065), is caused by mutations in the PMM2 gene. This gene encodes a phosphomannomutase that converts Man-6-phosphate to Man-1-phosphate. Mutations reduce the size of the GDPMan pool and produce insufficient lipid-linked oligosaccharide for full glycosylation. Other type-I CDGs are thought to be caused by defects in the biosynthesis of lipid-linked oligosaccharides in different steps. ${ }^{52}$ On the other hand, type-II CDGs are caused by alterations in the processing of $\mathrm{N}$-glycans on proteins, as described in the case of CDG-IIa above. CDG-IIc (OMIM 266265), which is caused by mutations in the Golgi GDP-fucose transporter rather than by mutations in enzymes, results in global fucosylation defects, profound mental retardation, failure to thrive, recurrent infections, and leukocytosis. CDG-IId has been observed in only one patient, who showed brain malformation, mental re- 
Table 1. Possible Muscular Dystrophies Caused by Abnormal Glycosylation of $\alpha$-Dystroglycan

\begin{tabular}{lll}
\hline \multicolumn{1}{c}{ Conditions } & & \multicolumn{1}{c}{ Gene } \\
\hline Muscle-eye-brain disease (MEB) & POMGnT1 & O-Mannosyl glycan GlcNActransferase \\
Fukuyama congenital muscular dystrophy (FCMD) & Fukutin & Putative glycosyltransferase \\
Walker-Warburg syndrome (WWS) (20\%) & POMT1 & Putative O-mannosyltransferase \\
MDC1C and Limb-girdle muscular dystrophy 2I (LGMD2I) & Fukutin-related protein (FKRP) & Putative glycosyltransferase \\
Myodystrophy $(m y d)$ mouse & Large & Putative glycosyltransferase \\
\hline
\end{tabular}

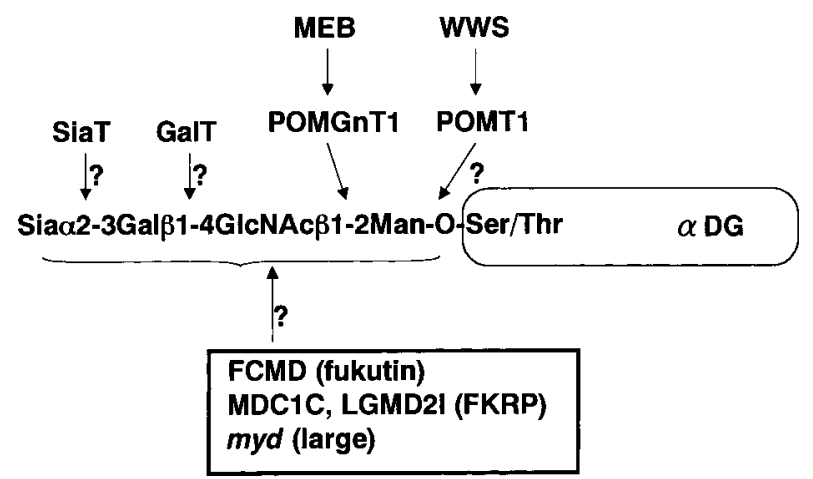

Fig. 4. Possible Defects of $O$-Mannosylglycosylation of $\alpha$-Dystroglycan in Muscular Dystrophy

Mutations in POMGnT1, POMT1, fukutin, FKRP and large cause defects in the glycosylation of $\alpha$-dystroglycan resulting in muscular dystrophy. The substrates of these putative enzymes, with the exception of POMGnT1, are largely unknown. POMT1 is thought to initiate the biosynthesis of $O$-mannosyl glycan. It is unclear whether other as yet uncharacterized forms of muscular dystrophy are caused by defects in galactosyltransferases (GalT) and sialyltransferases (SiaT).

tardation, myopathy, and blood clotting defects. This patient was found to have a $1 \mathrm{bp}$ insertion in the $\beta 4$ GalT I gene and galactosyltransferase activity (galactosylation of $N$-glycan) was reduced. ${ }^{53)}$ However, it is unclear whether the patient had abnormal muscle degeneration or defective glycosylation of $\alpha$-dystroglycan. Taken together, the CDG studies indicate that correct $N$-glycosylation of proteins is essential for normal development.

Perspectives In summary, hypoglycosylated $\alpha$-dystroglycan in the muscle membranes of MEB and FCMD patients and the myd mouse has greatly reduced affinities for laminin, neurexin and agrin. This suggests that defective glycosylation of $\alpha$-dystroglycan due to primary genetic defects of glycosyltransferases is the common denominator of muscle cell degeneration and abnormal brain structure of MEB, WWS, FCMD, MDC1C patients and the myd mouse (Table 1). In other words, interference in glycosylation of $\alpha$-dystroglycan may lead to a combination of abnormal muscle and brain phenotypes. However, the substrates of these enzymes, with the exception of POMGnT1, are largely unknown (Fig. 4). Identification and characterization of each enzyme will help to reveal the molecular pathomechanisms of congenital muscular dystrophies with brain malformation. Hypoglycosylation of $\alpha$-dystroglycan, a peripheral membrane glycoprotein expressed in muscle, brain and other tissues, is a common feature in these diseases. In the future, $\alpha$-dystroglycan may be a potential target of new glycotherapeutic strategies for muscular dystrophy as well as neuronal migration disorder. Future studies may also reveal that presently uncharacterized forms of muscular dystrophy are caused by defects in galactosyltransferases and/or sialyltransferases. A major challenge will be to integrate the forthcoming structural, cell biological, and genetic information to understand how $\alpha$-dystroglycan glycosylation contributes to muscular dystrophy and neuronal migration disorder.

Acknowledgements This study was supported by Research Grants for Nervous and Mental Disorders (14B-4) and Research on Psychiatric and Neurological Diseases and Mental Health from the Ministry of Health, Labour and Welfare of Japan, and by a Grant-in-Aid for Scientific Research on Priority Area (14082209) from the Ministry of Education, Culture, Sports, Science and Technology of Japan.

\section{REFERENCES AND NOTES}

1) Kobata A., Eur. J. Biochem., 209, 483-501 (1992).

2) Varki A., Glycobiology, 3, 97-130 (1993).

3) Emery A. E., Lancet, 359, 687-695 (2002).

4) Burton E. A., Davies K. E., Cell, 108, 5-8 (2002).

5) Strahl-Bolsinger S., Gentzsch M., Tanner W., Biochim. Biophys. Acta, 1426, 297-307 (1999).

6) Timpel C., Strahl-Bolsinger S., Ziegelbauer K., Ernst J. F., J. Biol. Chem., 273, 20837-20846 (1998).

7) Spiro R. G., Bhoyroo V. D., J. Biol. Chem., 255, 5347—5354 (1980).

8) Krusius T., Reinhold V. N., Margolis R. K., Margolis R. U., Biochem. $J ., 245,229-234$ (1987).

9) Krusius T., Finne J., Margolis R. K., Margolis R. U., J. Biol. Chem., 261, 8237-8242 (1986).

10) Finne J., Krusius T., Margolis R. K., Margolis R. U., J. Biol. Chem., 254, 10295-10300 (1979).

11) Chiba A., Matsumura K., Yamada H., Inazu T., Shimizu T., Kusunoki S., Kanazawa I., Kobata A., Endo T., J. Biol. Chem., 272, 2156-2162 (1997).

12) Sasaki T., Yamada H., Matsumura K., Shimizu T., Kobata A., Endo T., Biochim. Biophys. Acta, 1425, 599-606 (1998).

13) Ervasti J. M., Burwell A. L., Geissler A. L., J. Biol. Chem., 272, 22315-22321 (1997).

14) Yuen C. T., Chai W., Loveless R. W., Lawson A. M., Margolis R. U., Feizi T., J. Biol. Chem., 272, 8924-8931 (1997).

15) Chai W., Yuen C. T., Kogelberg H., Carruthers R. A., Margolis R. U., Feizi T., Lawson A. M., Eur. J. Biochem., 263, 879-888 (1999).

16) Smalheiser N. R., Haslam S. M., Sutton-Smith M., Morris H. R., Dell A., J. Biol. Chem., 273, 23698-23703 (1998).

17) Wing D. R., Rademacher T. W., Schmitz B., Schachner M., Dwek R. A., Biochem. Soc. Trans., 20, 386-390 (1992).

18) Endo T., Biochim. Biophys. Acta, 1473, 237-246 (1999).

19) Ibraghimov-Beskrovnaya O., Ervasti J. M., Leveille C. J., Slaughter C. A., Sernett S. W., Campbell K. P., Nature (London), 355, 696-702 (1992).

20) Winder S. J., Trends. Biochem. Sci., 26, 118-124 (2001).

21) Williamson R. A., Henry M. D., Daniels K. J., Hrstka R. F., Lee J. C., Sunada Y., Ibraghimov-Beskrovnaya O., Campbell K. P., Hum. Mol. Genet., 6, 831—841 (1997).

22) Cote P. D., Moukhles H., Lindenbaum M., Carbonetto S., Nat. Genet., 23, 338-342 (1999).

23) Cohn R. D., Henry M. D., Michele D. E., Barresi R., Saito F., Moore S. A., Flanagan J. D., Skwarchuk M. W., Robbins M. E., Mendell J. R., Williamson R. A., Campbell K. P., Cell, 110, 639-648 (2002). 
24) Moore S. A., Saito F., Chen J., Michele D. E., Henry M. D., Messing A., Cohn R. D., Ross-Barta S. E., Westra S., Williamson R. A., Hoshi T., Campbell K. P., Nature (London), 418, 422-425 (2002).

25) Saito F., Moore S. A., Barresi R., Henry M. D., Messing A., RossBarta S. E., Cohn R. D., Williamson R. A., Sluka K. A., Sherman D. L., Brophy P. J., Schmelzer J. D., Low P. A., Wrabetz L., Feltri M. L., Campbell K. P., Neuron, 38, 747-758 (2003).

26) Michele D. E., Barresi R., Kanagawa M., Saito F., Cohn R. D., Satz J. S., Dollar J., Nishino I., Kelley R. I., Somer H., Straub V., Mathews K. D., Moore S. A., Campbell K. P., Nature (London), 418, 417-422 (2002).

27) Takahashi S., Sasaki T., Manya H., Chiba Y., Yoshida A., Mizuno M., Ishida H., Ito F., Inazu T., Kotani N., Takasaki S., Takeuchi M., Endo T., Glycobiology, 11, 37-45 (2001).

28) Yoshida A., Kobayashi K., Manya H., Taniguchi K., Kano H., Mizuno M., Inazu T., Mitsuhashi H., Takahashi S., Takeuchi M., Herrmann R., Straub V., Talim B., Voit T., Topaloglu H., Toda T., Endo T., Dev. Cell, 1, 717-724 (2001).

29) Ioffe E., Stanley P., Proc. Natl. Acad. Sci. U.S.A., 91, 728-732 (1994).

30) Metzler M., Gertz A., Sarkar M., Schachter H., Schrader J. W., Marth J. D., Embo. J., 13, 2056-2065 (1994).

31) Wang Y., Tan J., Sutton-Smith M., Ditto D., Panico M., Campbell R. M., Varki N. M., Long J. M., Jaeken J., Levinson S. R., WynshawBoris A., Morris H. R., Le D., Dell A., Schachter H., Marth J. D., Glycobiology, 11, 1051-1070 (2001).

32) Tan J., Dunn J., Jaeken J., Schachter H., Am. J. Hum. Genet., 59, 810817 (1996).

33) Santavuori P., Somer H., Sainio K., Rapola J., Kruus S., Nikitin T., Ketonen L., Leisti J., Brain Dev., 11, 147-153 (1989).

34) Taniguchi K., Kobayashi K., Saito K., Yamanouchi H., Ohnuma A., Hayashi Y. K., Manya H., Jin D. K., Lee M., Parano E., Falsaperla R., Pavone P., Van Coster R., Talim B., Steinbrecher A., Straub V., Nishino I., Topaloglu H., Voit T., Endo T., Toda T., Hum. Mol. Genet., 12, 527-534 (2003).

35) Manya H., Sakai K., Kobayashi K., Taniguchi K., Kawakita M., Toda T., Endo T., Biochem. Biophys. Res. Commun., 306, 93-97 (2003).

36) Kano H., Kobayashi K., Herrmann R., Tachikawa M., Manya H., Nishino I., Nonaka I., Straub V., Talim B., Voit T., Topaloglu H., Endo T., Yoshikawa H., Toda T., Biochem. Biophys. Res. Commun., 291, $1283-1286$ (2002).

37) Fukuyama Y., Osawa M., Suzuki H., Brain Dev., 3, 1-29 (1981).

38) Dobyns W. B., Pagon R. A., Armstrong D., Curry C. J., Greenberg F., Grix A., Holmes L. B., Laxova R., Michels V. V., Robinow M., Am. J. Med. Genet., 32, 195-210 (1989).
39) Kobayashi K., Nakahori Y., Miyake M., Matsumura K., Kondo-Iida E., Nomura Y., Segawa M., Yoshioka M., Saito K., Osawa M., Hamano K., Sakakihara Y., Nonaka I., Nakagome Y., Kanazawa I., Nakamura Y., Tokunaga K., Toda T., Nature (London), 394, 388-392 (1998).

40) Aravind L., Koonin E. V., Curr. Biol., 9, R836-837 (1999).

41) Hayashi Y. K., Ogawa M., Tagawa K., Noguchi S., Ishihara T., Nonaka I., Arahata K., Neurology, 57, 115-121 (2001).

42) Takeda S., Kondo M., Sasaki J., Kurahashi H., Kano H., Arai K., Misaki K., Fukui T., Kobayashi K., Tachikawa M., Imamura M., Nakamura Y., Shimizu T., Murakami T., Sunada Y., Fujikado T., Matsumura K., Terashima T., Toda T., Hum. Mol. Genet., 12, 1449-1459 (2003).

43) Beltran-Valero De Bernabe D., Currier S., Steinbrecher A., Celli J., Van Beusekom E., Van Der Zwaag B., Kayserili H., Merlini L., Chitayat D., Dobyns W. B., Cormand B., Lehesjoki A. E., Cruces J., Voit T., Walsh C. A., Van Bokhoven H., Brunner H. G., Am. J. Hum. Genet., 71, 1033-1043 (2002).

44) Willer T., Amselgruber W., Deutzmann R., Strahl S., Glycobiology, 12, 771-783 (2002).

45) Martin-Blanco E., Garcia-Bellido A., Proc. Natl. Acad. Sci. U.S.A., 93, 6048-6052 (1996).

46) Brockington M., Blake D. J., Prandini P., Brown S. C., Torelli S., Benson M. A., Ponting C. P., Estournet B., Romero N. B., Mercuri E., Voit T., Sewry C. A., Guicheney P., Muntoni F., Am. J. Hum. Genet., 69, 1198-1209 (2001).

47) Brockington M., Yuva Y., Prandini P., Brown S. C., Torelli S., Benson M. A., Herrmann R., Anderson L. V., Bashir R., Burgunder J. M., Fallet S., Romero N., Fardeau M., Straub V., Storey G., Pollitt C., Richard I., Sewry C. A., Bushby K., Voit T., Blake D. J., Muntoni F., Hum. Mol. Genet., 10, 2851-2859 (2001).

48) Esapa C. T., Benson M. A., Schroder J. E., Martin-Rendon E., Brockington M., Brown S. C., Muntoni F., Kroger S., Blake D. J., Hum. Mol. Genet., 11, 3319-3331 (2002).

49) Grewal P. K., Holzfeind P. J., Bittner R. E., Hewitt J. E., Nat. Genet., 28, 151-154 (2001).

50) Holzfeind P. J., Grewal P. K., Reitsamer H. A., Kechvar J., Lassmann H., Hoeger H., Hewitt J. E., Bittner R. E., Hum. Mol. Genet., 11, 2673-2687 (2002).

51) Longman C., Brockington M., Torelli S., Jimenez-Mallebrera C., Kennedy C., Khalil N., Feng L., Saran R. K., Voit T., Merlini L., Sewry C. A., Brown S. C., Muntoni F., Hum. Mol. Genet., 12, 28532861 (2003).

52) Freeze H. H., Biochim. Biophys. Acta, 1573, 388-393 (2002).

53) Hansske B., Thiel C., Lubke T., Hasilik M., Honing S., Peters V., Heidemann P. H., Hoffmann G. F., Berger E. G., von Figura K., Korner C., J. Clin. Invest., 109, 725-733 (2002). 